\title{
Conocimiento y perfil de uso de la biblioteca Cochrane en médicos asistentes a un congreso de Medicina Interna
}

\author{
Luz M Letelier S'; María P Acuña S²; Paola Viviani G³; Ana P Díaz F; Sebastián Ewoldt P1; Solange Rivera M5*
}

Resumen: Introducción: las Revisiones Sistemáticas (RS) son herramientas para practicar Medicina Basada en la Evidencia. La Colaboración Cochrane genera RS, pero el conocimiento y uso de la Biblioteca Cochrane (BC) es heterogéneo. Nuestro objetivo fue describir el nivel de conocimiento y el perfil de uso de la BC entre asistentes a una Conferencia Mundial de Medicina Interna (WCIM). Método: estudio transversal vía encuesta electrónica entre asistentes al XXXI WCIM, recabando información demográfica de los participantes; su conocimiento, acceso y usos de la BC. Resultados: 413 asistentes aceptaron participar y 198 (47,9\%) de 24 países respondieron. 91,4\% eran Latinoamericanos, 50,5\% eran internistas. El conocimiento de la BC fue del $96,5 \%$. El 76\% de quienes respondieron usaban la BC al menos una vez al mes. No encontramos diferencias en la frecuencia de uso según edad o ámbito académico. Las principales razones para utilizar RS-BC fueron: toma de decisiones clínicas (67,6\%), actualización (64,2\%) y docencia (31,8\%); 46\% consideraron la BC muy útil para sus propósitos. Los $<35$ años utilizaban significativamente más la BC para guiar las decisiones clínicas que los $>35$ años (70\% vs $53 \%$, p: 0,017). Quienes consideraron muy útil la BC la utilizaron significativamente más para propósitos docentes (41\% vs. 24\%, p: 0,019) y toma de decisiones clínicas (79\% vs. 58\%, p: 0,003) que quienes la declararon como a veces útil o no útil. Hubo también diferencias estadísticamente significativas en la distribución del acceso a la $B C$ según región geográfica (p: 0,001). Conclusiones: encontramos un alto nivel de conocimiento de la BC entre los asistentes a WCIM. Los usuarios describen un uso frecuente para diversos propósitos, considerándola útil para estos.

Palabras clave: Biblioteca Cochrane; conocimiento; perfil de uso; Medicina Interna; Latinoamérica.

Abstract: Introduction: Systematic Reviews (SR) are tools for practicing Evidence Based Medicine. Cochrane Collaboration generates SRs, but awareness and uses of Cochrane SR, changes over time and across different countries and medical specialties. We aimed to describe awareness and user's profile of Cochrane Library (CL) SR among attendants to a World Conference of Internal Medicine (WCIM). Methods: Cross sectional online survey study among attendants to the XXXI WCIM asking about demographic information, awareness of, access to and uses of CL-SRs. Results: 413 attendants to WCIM volunteered to participate and 198 (47.9\%) from 24 countries replied; 91.4\% were from Latin-America. Mean age was 37 years and $50.5 \%$ were general internists or internal medicine subspecialists. Awareness of the $\mathrm{CL}$ was $96.5 \%$. The frequency of CL-SR use was at least once a month for $76 \%$ of responders. We found no difference in frequency of use according to age or academic setting. Main reasons to use CL-SR were: guide clinical decisions (67.6\%), personal update (64.2\%) and teaching (31.8\%); 46\% considered CL-SR very useful to their purposes. People <35y used CL-SR significantly more for guiding clinical decisions than those $>35 \mathrm{y}$ ( $70 \%$ vs $53 \%$, p: 0.017 ). Users who considered CL-SR very useful use it significantly more for teaching purposes ( $41 \%$ vs $24 \%$, p: 0.019 ) and for clinical decision-making (79\% vs 58\%, p: 0.003 ) than those declaring CL-SR as sometimes useful or not useful. Also there was statistically significant difference in distribution of access to CL by geographic region (p: 0.001). Conclusions: We found high awareness of CL-SRs among attendants to WCIM. Users describe frequent use, for several purposes and find it helpful for their purposes; however, access varied across regions.

Keywords: Cochrane Library; awareness ; Users profile; Internal Medicine; Latin America.

Fecha de envío: 8 de mayo de 2017 - Fecha de aceptación: 6 de noviembre de 2017

(1) Departamento de Medicina Interna, Facultad de Medicina, Pontificia Universidad Católica de Chile.

(2) Unidad de Infectología, Complejo Asistencial Dr. Sótero del Río.

(3) Departamento de Salud Pública, Facultad de Medicina, Pontificia Universidad Católica de Chile.

(4) Departamento de Enfermedades Respiratorias del Adulto, Pontificia Universidad Católica de Chile.

(5) Departamento de Medicina Familiar, Facultad de Medicina, Pontificia Universidad Católica de Chile.

*Autor de correspondencia: strivera@uc.cl. 


\section{Introducción}

Mantenerse al día en conocimientos de medicina es crucial para una atención de calidad a los pacientes. En particular, la Medicina Interna (MI) requiere de una amplia gama de conocimientos, permanentemente en renovación, por la aparición de nueva información; por esta razón, mantenerse actualizado requiere tiempo y habilidades (Rada \& Letelier, 2009). La salud basada en evidencia (SBE) ha desempeñado un papel importante en acercar la información científica a la toma de decisiones clínicas (Dawes et al., 2005), y reconoce las Revisiones Sistemáticas (RS) de ensayos clínicos aleatorios como la más alta jerarquía de evidencia para evaluar la eficacia y la seguridad de las intervenciones (Oxman et al., 1994). Por esto, las RS se consideran herramientas importantes y necesarias para la práctica de la medicina y, específicamente, de la medicina interna.

La Colaboración Cochrane (CC) es una organización internacional sin fines de lucro dedicada desde 1993 a generar RS de alta calidad, con el objetivo de acercar la investigación clínica a los profesionales de la salud y ayudarlos a tomar decisiones clínicas basadas en evidencia (El Dib et al, 2007; Sackett, 1994). Estas RS se difunden a través de la Biblioteca Cochrane (BC) (Villas et al., 2013). Una de las metas de la CC es mejorar el acceso y la difusión del conocimiento entre los responsables de la toma de decisiones en materia de salud (Friedrich, 2013). Para ello, han desarrollado iniciativas como la BC-Plus, que permite el libre acceso a las RS traducidas al español y portugués a todos los países latinoamericanos, en una alianza con la Organización Mundial de la Salud, para favorecer las políticas de salud basadas en la evidencia.

Sin embargo, a pesar de estos esfuerzos, el grado de conocimiento y la utilización de las RS de la BC no es lo deseable entre los clínicos y varía entre especialidades y región geográfica (O'Donnell, 2004). Varios estudios que describen el nivel de conocimiento de la BC muestran esta situación. Por ejemplo, una encuesta realizada a internistas canadienses en 1997 reportó que el 5\% utilizó la BC como fuente de información de manera regular (McAlister, 1999). El mismo año, otra encuesta entre 302 médicos generalistas del Reino Unido encontró que el 60\% desconocía la BC; el 31\% la conocía pero no la utilizaba, y solo el 5\% la leía (McColl et al., 1998). En el 2009, un sondeo telefónico entre 573 médicos de Croacia informó un $34 \%$ de conocimiento de la BC, pero con diferencias estadísticas entre médicos de familia y médicos de hospitales universitarios (20\% y 55\%, respectivamente, $p<0,001$ ) (Novak et al., 2010). Más recientemente, en China un 4\% de 444 cardiólogos e internistas encuestados eran conscientes de la BC como una fuente de RS (Wang et al., 2011). Los médicos noruegos de todas las especialidades fueron entrevistados con respecto a las fuentes de información para la práctica clínica. El 27\% consideraba la BC como una fuente de información (Ulvenes et al., 2009). Otro estudio realizado a 141 médicos de familia jordanos mostró resultados similares: el 60,3\% ignoraba la existencia de la BC, el 26,2\% la conocía pero no la utilizaba, el 9,2\% leía las RS de la BC y solo el 4,3\% las utilizaba para la toma de decisiones clínicas (Barghouti et al., 2009).

En un hospital universitario de Dinamarca, la BC fue la fuente de información menos consultada, donde un 49\% de los médicos participantes declararon nunca haberla consultado (Oliveri et al., 2004). En Malasia, el conocimiento de la BC y sus RS fue de $32,5 \%$ entre 46 médicos de familia (Chang \& Teng, 2005). De modo similar, en Arabia Saudita, de 650 médicos que trabajaban en los centros de atención primaria del Ministerio de Salud de Riyadh, el 14,4\% conocía la BC (Al-Ansary \& Khoja, 2002). Entre los onco-radiólogos de Nueva Zelandia y Australia, una minoría, el 28\% utiliza la BC (Veness et al., 2003). En relación a América Latina, hay escasa información sobre el conocimiento y uso de la BC. Un estudio entre investigadores de países latinoamericanos mostró que solo un 4.9\% reporta usarla (Ospina et al., 2005).

En un contexto clínico más globalizado y con la mayor difusión que ha tenido la SBE, podría esperarse que el nivel de conocimiento y la utilización de la BC aumenten. No obstante algunos estudios no apoyan este supuesto, por ejemplo, una encuesta entre médicos y residentes de diferentes especialidades en Jordania reveló que el $48 \%$ no conocía la BC, $27 \%$ eran conscientes de su existencia pero no la utilizaban y que solo el $5 \%$ la usaba para la toma de decisiones clínicas (Al Omari et al., 2009).

Considerando la relevancia de la utilización de las RS de la BC en la práctica clínica de la Medicina Interna, nos pareció necesario aproximarnos al nivel de conocimiento actual de la BC y sus RS entre los profesionales de la especialidad y describir el perfil de los usuarios en términos de utilización, modo de acceso, frecuencia de uso y percepción de utilidad; con énfasis en América Latina.

\section{Objetivo}

Identificar el nivel de conocimiento de las RS de la BC entre los asistentes a un Congreso Mundial de Medicina Interna y describir el perfil de los usuarios de la BC.

\section{Métodos}

Estudio transversal mediante encuesta en línea, vía internet.

Desarrollamos una encuesta electrónica de 11 preguntas en inglés, el idioma oficial del evento. El cuestionario consistió en 5 preguntas para caracterizar a la población, 2 para identificar el nivel de conocimiento de la BC y 4 para describir el perfil de los usuarios 
incluyendo: modo de acceso, la frecuencia de uso, el propósito de usarlo y la percepción de utilidad de acuerdo al propósito de su uso.

La población comprendió a los participantes del XXXI World Conference of Internal Medicine (WCIM) realizado en Chile. Este Congreso se realiza cada dos años y reúne a médicos internistas de todo el mundo.

Se reclutó aleatoriamente a voluntarios para responder la encuesta. El equipo de investigación invitó personalmente a los asistentes a participar. Si aceptaban se les solicitaba su correo electrónico para ser contactados posteriormente. El primer correo electrónico fue enviado durante las primeras 72 horas después del contacto personal. A los participantes que no respondieron después del primer correo electrónico, se les envió 3 recordatorios durante un período de 12 semanas. Las respuestas fueron recogidas por un programa de encuestas en línea y tabuladas por los investigadores.

La edad se expresa como media \pm desviación estándar. Las variables categóricas se expresan como frecuencia y porcentaje. Los datos categóricos: grupo etario, setting, región o frecuencia de uso se analizaron mediante la prueba de Chi cuadrado o prueba exacta de Fisher.

El punto de corte etario para las comparaciones fue de 35 años, dado que los sujetos bajo esa edad pueden considerarse"nativos digitales"(Prensky, 2001). Definimos "setting académico" como aquellos que reportaron ser estudiantes de medicina o residentes, más aquellos médicos que reportaron trabajar en un centro universitario u otro ambiente académico.

\section{Resultados}

Reclutamos 413 asistentes, que consintieron a dar su dirección de correo electrónico; todos ellos recibieron la encuesta en línea y los recordatorios, según correspondiera. Se obtuvieron 198 respuestas $(47,9 \%)$.

\section{Características de los participantes:}

La edad media de quienes respondieron fue de 37 años (DE \pm 12,3), 117 (59,1\%), eran hombres y provenían de 24 países, en su mayoría de América Latina (91,4\%). 116 (58,6\%) eran chilenos, 65 $(32,8 \%)$ de otros países latinoamericanos y 17 (8,6\%) de países no latinoamericanos (Figura 1a). 100 (50.5\%) eran internistas generales o sub-especialistas de Ml; 53 (26,8\%) eran residentes, 33 (16,7\%) estudiantes de medicina y $12(6,1 \%)$ médicos de otras especialidades $\mathrm{u}$ otros profesionales de la salud (Figura $1 \mathrm{~b}$ ).
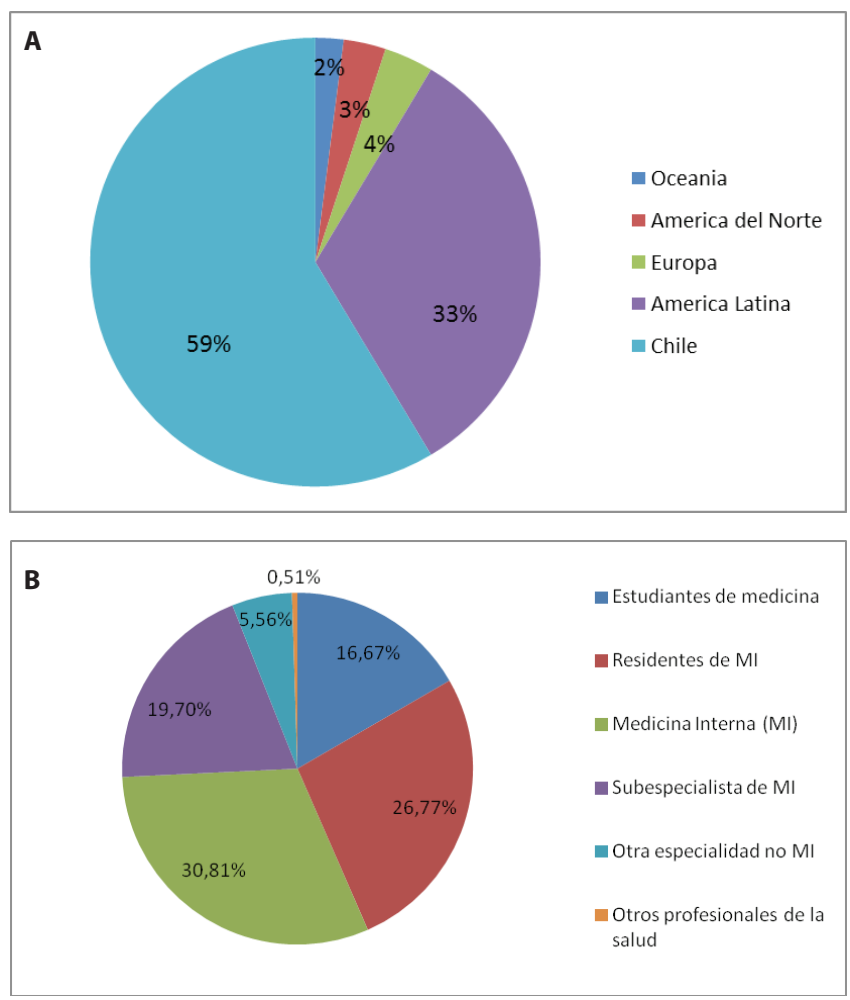

Figura 1: Características de la población encuestada. A) Muestra la distribución geográfica porcentual de los participantes que respondieron la encuesta: 116 eran chilenos, 65 de otros países latinoamericanos y 17 de países no latinoamericanos. B) Describe la actividad o etapa profesional de los participantes que respondieron la encuesta: 100 eran internistas generales o sub-especialistas de $\mathrm{Ml} ; 53$ eran residentes, 33 estudiantes de medicina y 12 médicos de otras especialidades u otros profesionales de la salud.

En cuanto al lugar de trabajo principal, 155 (78,3\%) trabajaban mayoritariamente en el hospital y $47(23,7 \%)$ predominantemente en actividad ambulatoria; 121 (61,1\%) se desempeñaban en un setting académico.

\section{Nivel de conocimiento de la Biblioteca Cochrane:}

191 (96,5\%) de los encuestados declararon haber consultado la BC como fuente de RS al menos una vez y 77 de ellos (38,9\% del total) declara utilizar además otras fuentes para obtener RS. Las razones de los 7 (3,5\%) que no utilizaron la BC, fueron: 2 no tenían acceso regular, 1 no sabía de su existencia, otros 3 no usaban ninguna fuente de RS y 1 no declaró razones.

\section{Perfil de los usuarios de la Biblioteca Cochrane:}

De los respondedores, 176 personas declararon la frecuencia de uso de la BC durante el año precedente. Una persona $(0,6 \%)$ refirió consultar la $\mathrm{BC}$ al menos una vez al día, 37 (21\%) la consulta al menos una vez a la semana, 96 (54,5\%) al menos una vez al mes, 
$28(15,9 \%)$ dos veces al año y $14(8 \%)$ solo una vez al año. Es decir, que entre los usuarios de la BC, un $76.1 \%$ la usa al menos una vez al mes. No hubo diferencias estadísticas en la frecuencia de uso de BC entre los grupos etarios (75,7\% para $\geq 35$ años vs $76,5 \%$ para $<35$ años, $p=0,903)$. De los usuarios inmersos en un ambiente académico el 77,8\% usó la BC al menos una vez al mes, comparado con el 73,5\% de aquellos no relacionados a un ambiente académico, sin diferencia estadísticamente significativa $(p=0,52)$. Tampoco encontramos diferencia en la frecuencia de uso al comparar por género o por región geográfica (América Latina vs. otros países). Respecto del propósito al usar las $\mathrm{RS}$ de la $\mathrm{BC}$, el 70.5\% reportó más de una razón, siendo las principales: toma de decisiones clínicas (119 participantes, 67,6\%), actualización continua (113 participantes, 64,2\%), fines de docencia (56 participantes, 31,8\%), uso en investigación (47 participantes, $26,7 \%$ ), formulación de políticas de salud (11 participantes, 6,3\%). Los respondedores menores de 35 años usaron las RS de la BC significativamente más a menudo para guiar las decisiones clínicas que los usuarios de 35 años y más (70\% vs 53\%, $\mathrm{p}=0.017$ ).

Ochenta y un respondedores (46\%) consideraron que las RS de la BC eran muy útiles y 93 (52.8\%), las consideraron a veces útiles en relación con su propósito de uso. Dos personas (1\%) declararon que no eran útiles para sus propósitos. Los usuarios que consideraron la BC muy útil, la utilizaban mucho más para propósitos docentes ( $41 \%$ vs. $24 \%, p=0.019$ ) y para toma de decisiones clínicas (79\% vs $58 \%, p=0.003$ ) que quienes las consideraban como a veces útil o no útil. Aquellos que la reportaron como muy útil tienen 3.08 veces más posibilidades de usarla al menos una vez al mes, respecto a quienes la consideraron a veces útil o no útil (IC95\% OR: 1.29-6.13).

\section{Acceso a la BC:}

El acceso a la BC ocurre a través de suscripción institucional para 79 respondedores (45\%), acceso gratuito a través de BC Plus para 67 respondedores (38\%), a través de un colega 25 respondedores $(14,2 \%)$ y 5 respondedores (2,8\%) pagan su acceso personal.

Al analizar por región geográfica, encontramos una diferencia estadísticamente significativa ( $p=0,001$ ) en relación a la distribución del acceso a la BC: $41 \%$ de los latinoamericanos obtienen acceso gratuito a través de CL Plus versus un 7\% de los usuarios no latinoamericanos. Respecto al acceso a través de suscripción institucional, alcanza un 40\% para latinoamericanos y un 93\% para los respondedores no latinoamericanos (Figura 2).
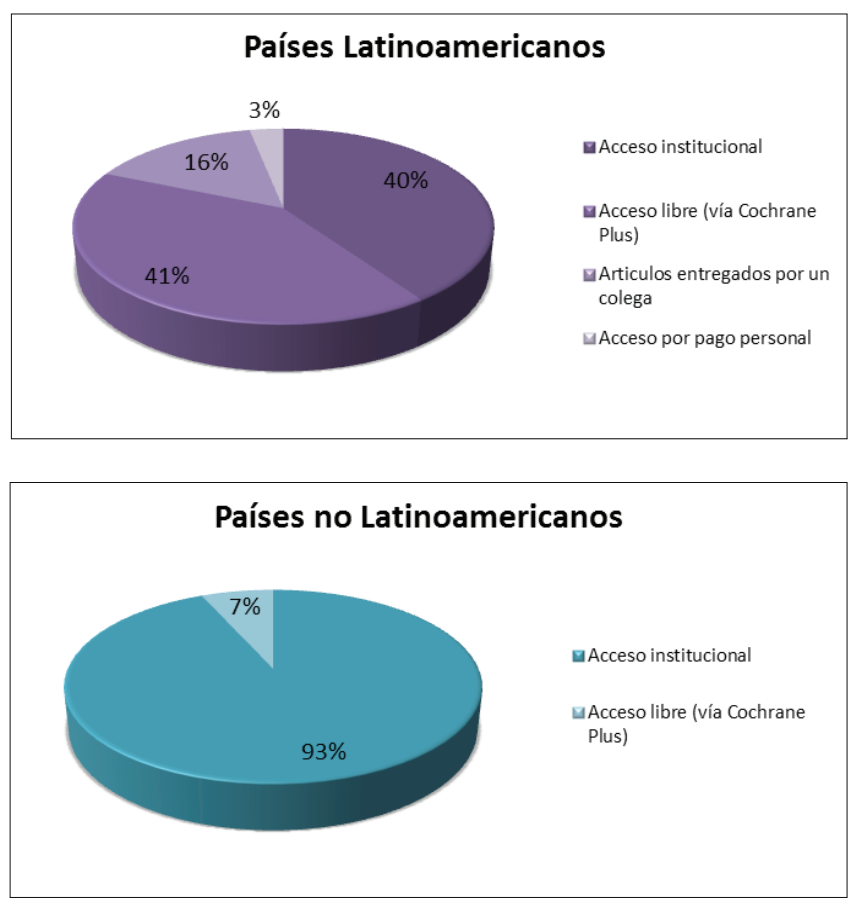

Figura 2: Acceso a la Biblioteca Cochrane. Describe la distribución porcentual de la vía de acceso a la BC entre los participantes de países latinoamericanos comparado con las vías de acceso utilizadas por los participantes no latinoamericanos.

\section{Discusión}

El nivel de conocimiento de la $B C$ es muy alto entre el grupo de asistentes al XXXI World Conference of Internal Medicine. La gran mayoría de quienes respondieron, consideraron que la BC era su principal fuente de RS, la usaban muy a menudo y la percibían como a veces útil o muy útil para sus propósitos.

Entre los encuestados no hubo diferencias estadísticamente significativas en la frecuencia de uso de la BC cuando se compararon por grupo etario o setting académico. En cambio, sí hubo diferencias estadísticamente significativas respecto al propósito de la BC al comparar por grupo etario: las personas más jóvenes usaban las RSs de la BC más a menudo para tomar decisiones clínicas.

Los usuarios que consideraron la BC como muy útil, tenían mayor probabilidad (OR 3.08 (IC95\% 1.29-6.13) de darle un uso clínico o docente, que quienes la consideraron a veces útil y tienen además una mayor probabilidad de usarla al menos una vez al mes. 
El análisis de la vía de acceso a la BC entre las regiones geográficas revela otra diferencia interesante: para los usuarios no latinoamericanos prevalece el acceso institucional, mientras que para los usuarios latinoamericanos el acceso libre a través de BC Plus es tan importante como el institucional. Esta diferencia podría explicarse en parte porque BC Plus contiene RS traducidas al español y posiblemente por un menor número de instituciones latinoamericanas con suscripciones a $\mathrm{BC}$ pagadas.

Cabe destacar el alto porcentaje de personas en nuestro estudio que declararon conocer y utilizar con frecuencia la BC, en relación a lo reportado en la literatura. Las posibles explicaciones de esta diferencia podrían relacionarse con el predominio de internistas de nuestra muestra, donde el uso de RS es habitual. Adicionalmente, los temas de Ml están bien representados en los contenidos de las $\mathrm{RS}$ de la $\mathrm{BC}$, lo que podría explicar que a un alto porcentaje de los encuestados, las RS de la BC les resultan muy útiles para tomar decisiones clínicas. Otro factor involucrado es el creciente desarrollo tecnológico que ha permitido mayor a acceso a internet, especialmente en la población más joven.

La población de nuestro estudio se desempeña en su mayoría en un setting académico, en el cual se ha reportado mayor uso de RS, a modo de ejemplo: un estudio reciente realizado entre médicos académicos altamente seleccionados de las capitales de la Unión Europea y los Estados Independientes de la Comunidad reveló un $99 \%$ y un $57 \%$ de conocimiento de la BC, respectivamente (Van der Voort et al., 2012). Nuestro estudio comprende una población seleccionada de médicos, dado que asistió voluntariamente a una conferencia mundial, denotando interés en la formación continua, lo que podría sobrestimar el uso de RS en otros grupos médicos.

Por último, es posible que solo aquellos encuestados que conocían la $\mathrm{BC}$ respondieran nuestra encuesta; si esto fuera la explicación y suponiendo el peor escenario, es decir que todos los asistentes contactados que no respondieron la encuesta no conocían la BC, aun así, un 46\% (191/413) de todos los asistentes contactados sí la conocerían, lo que representa una proporción superior a la anteriormente informada para médicos internistas (McAlister, 1999, Novak et al., 2010 y Chang \& Teng, 2005) y mucho más alta de lo reportado en Latinoamérica (Ospina et al., 2005).

Dentro de las limitaciones de este estudio, cabe señalar la tasa de respuesta de $48 \%$, aunque varios autores consideran que es una tasa de respuesta aceptable para encuestas en línea (Cook et al., 2000; De Marchis, 2012). Otras limitaciones son la falta de representación de países no latinoamericanos y el número sorprendentemente bajo de respondedores que declara no conocer el BC y no utilizar RS, lo que nos impidió comparar aquellos que la conocen y / o utilizan con aquellos que no lo hacen.
Este estudio contribuye a una mejor comprensión del perfil de los usuarios de la BC en términos de frecuencia de uso, propósito para su uso y modo de acceso en un grupo de clínicos relacionados con la Medicina Interna y principalmente latinoamericanos. Esto es de particular interés, ya que la información sobre el uso de la $B C$ en América Latina es escasa y su penetración en la región se ha considerado muy baja (Al-Ansary \& Khoja., 2002) debido a la sub-representación de estudios latinoamericanos o artículos escritos en español en la BC (Williams et al., 2008).

Esta información podría utilizarse para comparar el nivel de conocimiento y el perfil de los usuarios con otras especialidades médicas, otros profesionales de la salud, así como entre diferentes regiones geográficas.

\section{Referencias}

Al-Ansary L \& Khoja T (2002). The place of evidence-based medicine among primary health care physicians in Riyadh region, Saudi Arabia. Fam Pract. 19(5):537-542.

Al Omari M, Khader Y, Jadallah K, Dauod AS, Al-Shdifat A \& Khasawneh N (2009). Evidence-based medicine among hospital doctors in Jordan: awareness, attitude and practice. J Eval Clin Pract. 15(6):1137-1141.

Barghouti F, Halaseh L, Said T, Mousa A \& Dabdoub A (2009). Evidence-based medicine among Jordanian family physicians: awareness, attitude, and knowledge. Can Fam Physician 55(7):e6-13.

Chan G \& Teng C. (2005). Primary care doctors' perceptions towards evidence-based medicine in Melaka State: a questionnaire study. Med J Malaysia 60(2):130-133.

Cook C, Heath F \&Thompson R. (2000). A meta-analysis of response rates In web- or internet-based surveys. Educational and Psychological Measurement 60 (6): 821-836.

Dawes M, Summerskill W, Glasziou P, Cartabellotta A, Martin J, Hopayian K, Porzsolt F, Burls A \& Osborne J; Second International Conference of Evidence-Based Health Care Teachers and Developers (2005). Sicily statement on evidence-based practice. BMC Med Educ. 5 (1):1.

De Marchis G. (2012). La validez externa de las encuestas en la web. Amenazas y su control. Estudios sobre el Mensaje Periodístico 18 263-272.

El Dib R, Atallah A \& Andriolo R. (2007) Mapping the Cochrane evidence for decision making in health care. J Eval Clin Pract 13(4):689-692. 
Friedrich M (2013). The Cochrane Collaboration turns 20: assessing the evidence to inform clinical care. JAMA 309 (18): 1881-1882.

McAlister F, Graham I, Karr G \& Laupacis A (1999). Evidence-based medicine and the practicing clinician. J Gen Intern Med 14(4):236-42.

McColl A, Smith H, White P \& Field J (1998). General practitioner's perceptions of the route to evidence based medicine: a questionnaire survey. BMJ 316 (7128) 361-365.

Novak K, Mirić D, Jurin A, Vukojević K, Aljinović J, Čarić A, Marinović Guić $M$, Poljičanin $A$, Košta $V$, Rako $D$, Marušić $A$, Marušić $M$ \& Puljak L (2010). Awareness and use of evidence-based medicine databases and Cochrane Library among physicians in Croatia. Croat Med J 51(2):157-64.

O'Donnell C (2004). Attitudes and knowledge of primary care professionals towards evidence-based practice: a postal survey. $J$ Eval Clin Pract 10(2):197-205.

Oliveri R, Gluud C \&Wille-Jørgensen P (2004). Hospital doctors' self-rated skills in and use of evidence-based medicine - a questionnaire survey. J Eval Clin Pract 10(2):219-226.

Ospina E, Reveiz L Herault L, Cardona A (2005). The use of bibliographic databases by Spanish-speaking Latin American biomedical researchers: a cross-sectional study. Rev Panam Salud Publica 17(4):230-236.

Oxman A, Cook D \& Guyatt G (1994). Users' guides to the medical literature. VI. How to use an overview. Evidence-Based Medicine Working Group. JAMA 272(17):1367-71.

Prensky M. (2001). Digital Natives, Digital Immigrants. On the Horizon. NCB University Press 9 (5).
Rada G \& Letelier L. (2009). How can an internist keep updated in the XXIst century? Rev Med Chil. 137(5):701-8.

Sackett D (1994). The Cochrane Collaboration. ACP JClub 120 (3):A11. Ulvenes L, Aasland O, Nylenna M \& Kristiansen I (2009). Norwegian physicians' knowledge of and opinions about evidence-based medicine: cross-sectional study. PLoS One 4(11):e7828.

Van der Voort C, Swenne C, van der Hoorn-van Velthoven C \& Belt J. (2012) Online medical literature consultation habits of academic teaching physicians in the EU and CIS countries: a cross-sectional study. PLoS One 7(11):e44302.

Veness M, Rikard-Bell G \& Ward J (2003). Views of Australian and New Zealand radiation oncologists and registrars about evidence-based medicine and their access to Internet based sources of evidence. Australas Radiol 47(4):409-415.

Villas P, Spagnuolo R, Kamegasawa A, Braz L, Polachini do Valle A, Jorge E, Yoo H, Cataneo A, Corrêa I, Fukushima F, do Nascimento P, Módolo N, Teixeira M, de Oliveira E, Daher S \& El Dib R. (2013). Systematic reviews showed insufficient evidence for clinical practice in 2004: what about 2011? The next appeal for the evidence-based medicine age. J Eval Clin Pract. 19(4):633-637.

Wang Wei-Zhong, Tang Jin-Ling, Hu Yong-Hua, An Jian-Gang, Wang Yan-Ling, Ren Zhong-He, Zhang Hong \& Sian Griffiths (2011). Gap Between Evidence And Physicians' Knowledge And Practice Regarding Hypertension And Its Drug Treatment: A Survey In A Chinese City. Chin Med J (Engl) 124(8):1235-1241.

Williams J, Bórquez A \& Basáñez M (2008). Hispanic Latin America, Spain and the Spanish-speaking Caribbean: a rich source of reference material for public health, epidemiology and tropical medicine. Emerg Themes Epidemiol 5:17. 